\title{
Quantum Information: Quantum registers hit the right wavelength
}

Siddharth Dhomkar $^{1 *}$ and John J.L. Morton ${ }^{1,2 \dagger}$

${ }^{1}$ London Centre for Nanotechnology, University College London, London, WC1H OAH, UK

${ }^{2}$ Department of Electronic \& Electrical Engineering, University College London, London, WC1E 7JE, UK

"s.dhomkar@ucl.ac.uk, †jjl.morton@ucl.ac.uk

\section{Controlling nuclear spins coupled to an electron spin in silicon carbide has enabled development of a 'quantum register' interfaced with telecom photons, leading to the possibility of distant transport of quantum information.}

The control of impurities in optical fibres has enabled the ultra-low-loss transmission of near infrared (NIR) light that underpins our ability to share vast quantities of data around the world. Such optical communication channels are already being upgraded to possess in-built security enshrined by the laws of quantum mechanics, but only over short point-to-point connections. Quantum interfaces that can connect light to matter-based registers of quantum bits (used to generate and purify quantum entanglement among distant sites) are required to allow fundamentally optical communication over the long distances required for widespread impact. In this context, carefully selected optical impurities can play a role ${ }^{1}$. Writing in this issue of Nature Materials, Alexandre Bourassa and colleagues ${ }^{2}$ demonstrate exceptional control of a register of electron and nuclear spins associated with the divacancy (VV) defect in silicon carbide (SiC), firmly establishing it as an attractive system for the development of optical quantum networks.

An early quantum interface front-runner has been based on the nitrogen-vacancy (NV) colour centre in diamond; this atomic defect contains an electron spin, which can interact with a register of nearby nuclear spins in the lattice and has a suitable spin-selective optical transition around $637 \mathrm{~nm}$ enabling coupling to light. Still, the inability of NV centres to emit in the NIR band - where optical fibre transmission is high combined with the relative immaturity of advanced fabrication in diamond continues to motivate the search for the ideal quantum interface.

Thanks largely to its superior performance in high power electronics, $\mathrm{SiC}$ has seen rapid development with established device fabrication, meeting the exacting standards of the semiconductor industry ${ }^{3}$. SiC accommodates a variety of lattice defects emitting in the NIR spectral region, of which, VV is arguably the most promising ${ }^{4}$ (with emission at around $1100 \mathrm{~nm}$ ). The electron spin of the defect acts as the key matterbased degree of freedom, which can represent a quantum bit and couple to an optical transition in the NIR. In addition to these lattice defects, the host material has trace quantities of nuclear ${ }^{13} \mathrm{C}$ and ${ }^{29} \mathrm{Si}$ isotopes that possess a nuclear spin (Figure 1), but their presence is a double-edged sword. On the one hand, nuclear spins offer the potential for a versatile quantum register able to store quantum information for long times ${ }^{5}$ or perform basic quantum algorithms to increase the fidelity of remotely entangled pairs through a process known as distillation ${ }^{5}$; on the other hand, unintentional electron-nuclear interactions can result in rapid corruption of the quantum information stored in the electron spin, manifested as a reduced spin coherence lifetime. Having two dissimilar nuclear species in $\mathrm{SiC}$ assists in partial suppression of such detrimental effects - compared, for example, to materials like diamond or silicon - but is insufficient to completely eradicate them. By tuning nuclear spin concentration, Bourassa and coworkers ${ }^{2}$ reveal that a delicate balancing act is indeed achievable to harness power of nearby nuclear spins for well-controlled spin register. 
To demonstrate a quantum register in $\mathrm{SiC}$ with NIR coupling, Alexandre Bourassa and colleagues ${ }^{2}$ begin with a SiC crystal of natural nuclear spin abundance (about $1 \%{ }^{13} \mathrm{C}$ and $5 \%{ }^{29} \mathrm{Si}$ ) and identify a ${ }^{29} \mathrm{Si}$ spin in the vicinity of the VV defect that is strongly coupled to its electron spin. Using a protocol originally devised for $\mathrm{NV}$ centres in diamond ${ }^{6}$, they transfer the polarisation from the electron spin to the nuclear spin to achieve a high degree of nuclear polarisation. After initialising both spins in the register, they then apply a series of resonant radio frequency (RF) excitations to entangle both spins (Figure 1), exploiting a control sequence previously tested using ensembles of SiC defects ${ }^{7}$.

Although quantum registers possessing strong electron-nuclear couplings are beneficial for tasks that require fast quantum operations, stronger communication between the spins results in an unintended backaction on one spin as a by-product of an operation being performed on another. Hence, the authors study also single VV spins in isotopically engineered $\mathrm{SiC}$ with reduced nuclear spin content. Isotope engineering allows precise coupling to more distant nuclear spins that interact weakly with the colour centre while also enabling long electron spin lifetimes, up to about $15 \mathrm{~ms}$. These results show that tuning the nuclear spin concentration is a practical way to obtain quantum registers with coupling strengths and coherence times that can be optimised for different applications. Moreover, building upon the prior knowledge on initialisation and readout of the electron spins by means of resonant excitation ${ }^{4}$, Alexandre Bourassa and co-workers ${ }^{2}$ corroborate that quantum operations can definitely be performed with minimal error rates, attaining quantum information processing with gate fidelities over $99.98 \%$ within the register.

Beyond doubt, these results establish VVs in $\mathrm{SiC}$ as a practically viable material platform for quantum technologies and particularly as the nodes of a quantum network connected by NIR optical channels. Nevertheless, there are several hurdles to be overcome before such VV-based quantum interfaces become a reality. Entangling distant qubit registers using light is a clear subsequent milestone but this will require addressing the challenge that photon collection efficiency is low, even when using advanced IR detectors. Adding to the difficulty is the fact that only about $7 \%$ of the total number of emitted photons are truly indistinguishable ${ }^{4}$ due to phonon-induced broadening of the emission spectrum, making entanglement based on photon interference ${ }^{8}$ extremely challenging. Low photon counts have also impeded the progress towards 'single shot' spin readout in $\mathrm{SiC}$, such that measurements require multiple averages. Here, the nuclear spin registers realised by Alexandre Bourassa and colleagues. ${ }^{2}$ could be used for quantum nondemolition measurement protocols ${ }^{9}$ to enhance readout fidelity. Addressing these challenges is likely to require the use of photonic structures ${ }^{10,11}$ tailor-made for NIR and designed to enhance both the emission and collection of light.

In principle $\mathrm{SiC}$ can cope well with the nanofabrication and material engineering needed to enhance photon coupling, which implies that the future is bright for VV-based quantum devices. Some impurities at least, are worth keeping. 


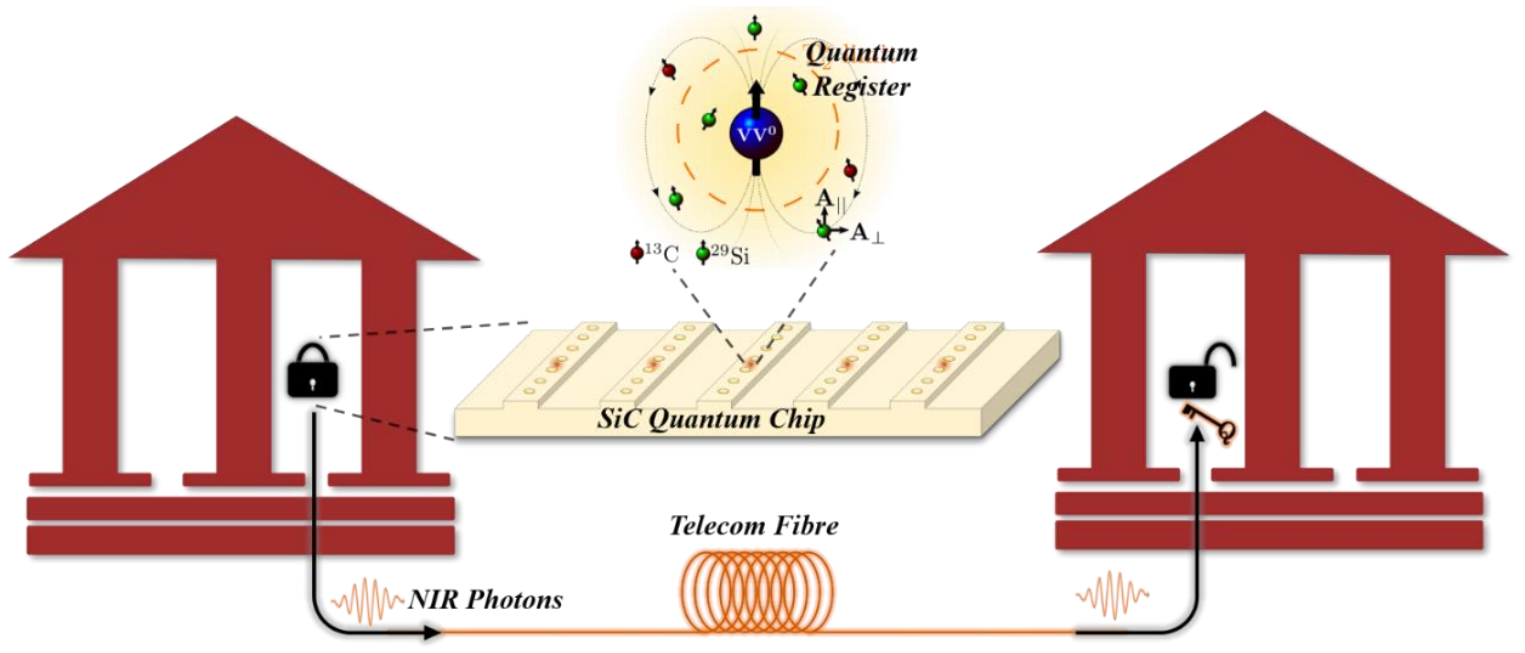

Figure 1. Long-distance quantum communication with quantum registers that emit NIR photons. Quantum registers ${ }^{2}$ comprising impurity spins entangled with surrounding nuclear spins in SiC can process quantum information at one end of the network, transporting it to the other via conventional telecom fibres. Emission of NIR photons by these spin defects renders efficient information transmission, bringing us closer to the possibility of virtually unhackable 'quantum-encrypted' data sharing.

1. Awschalom, D. D., Hanson, R., Wrachtrup, J. \& Zhou, B. B. Quantum technologies with optically interfaced solid-state spins. Nat. Photonics 12, 516-527 (2018).

2. Bourassa, A. et al. Entanglement and control of single nuclear spins in isotopically engineered silicon carbide. Nat. Mater. (2020).

3. Kimoto, T. Material science and device physics in SiC technology for high-voltage power devices. Jpn. J. Appl. Phys. 54, 040103 (2015).

4. Christle, D. J. et al. Isolated spin qubits in SiC with a high-fidelity infrared spin-to-photon interface. Phys. Rev. X 7, 021046 (2017).

5. Kalb, N. et al. Entanglement distillation between solid-state quantum network nodes. Science 356, 928-932 (2017).

6. Dutt, M. V. G. et al. Quantum Register Based on Individual Electronic and Nuclear Spin Qubits in Diamond. Science 316, 1312-1316 (2007).

7. Klimov, P. V., Falk, A. L., Christle, D. J., Dobrovitski, V. V. \& Awschalom, D. D. Quantum entanglement at ambient conditions in a macroscopic solid-state spin ensemble. Sci. $A d v . \mathbf{1}$, e1501015 (2015).

8. Bernien, H. et al. Heralded entanglement between solid-state qubits separated by three metres. Nature 497, 86-90 (2013).

9. Robledo, L. et al. High-fidelity projective read-out of a solid-state spin quantum register. Nature 477, 574-578 (2011).

10. Lukin, D. M. et al. 4H-silicon-carbide-on-insulator for integrated quantum and nonlinear photonics. Nat. Photonics 14, 330-334 (2019).

11. Crook, A. L. et al. Purcell enhancement of a single silicon carbide color center with coherent spin control. Nano Lett. 20, 3427-3434 (2020). 